\title{
A case report of laryngeal amyloidosis in a patient with stridor
}

\author{
Dr Sanjay Patel, Mr Venkat Srinivasan
}

\section{Wirral University Teaching Hospital}

\section{Introduction}

Laryngeal amyloidosis is a rare condition. In the majority of cases it is characterised by the deposition of amyloid protein isolated to the larynx but can also be a manifestation of the systemic form. Histochemically amyloidosis can be distinguished into light chain (LA) amyloidosis and secondary (AA) amyloidosis.

Isolated laryngeal amyloidosis represents $<1 \%$ of benign lesions of the larynx. It is the most common site for isolated amyloid deposits to occur in the head and neck. The presentation is characterised by an insidious onset of aerodigestive symtptoms, most commonly hoarseness or dysphonia. Episodic choking, dysphagia, dyspnoea and odynophagia are also reported in the literature. Stridor is a rarer symptom of laryngeal amyloidosis. ${ }^{1}$

\section{Objective and Methods}

We present a rare presentation of laryngeal amyloidosis with stridor to highlight its varied clinical course and presentation.

\section{Results}

A 79 year old ex-smoker with a past medical history of stroke and atrial fibrillation on warfarin presented to the ENT clinic with hoarseness. He was referred with a 3 month history of worsening hoarseness on a protracted history of hoarseness for 2-3 years. On presentation for microlaryngoscopy + biopsy he was noted to be in stridor. MRI demonstrated a right sided laryngeal lesion (Figure 1).

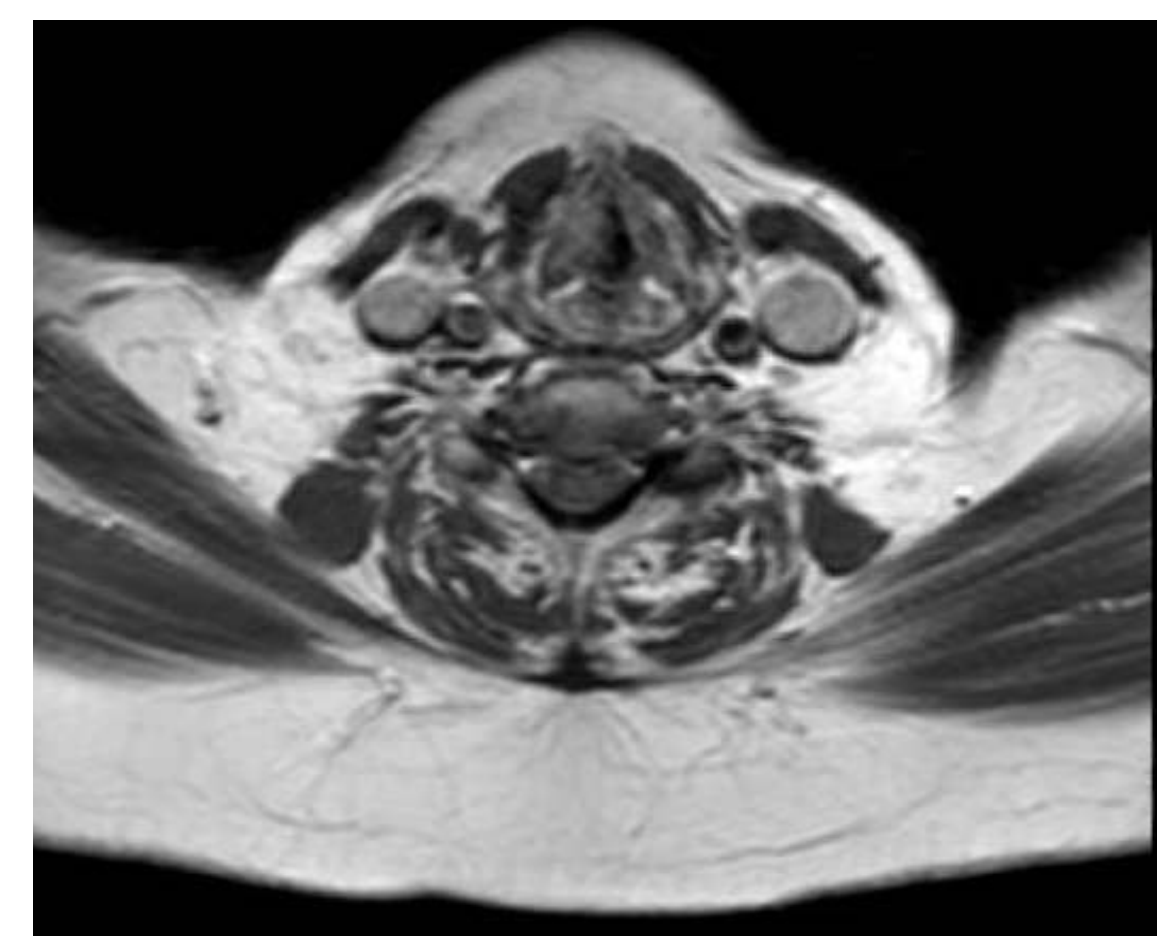

Figure 1

demonstrating MRI of the right laryngeal lesion

Microlaryngoscopy revealed a right sided proliferative lesion mainly affecting the subglottis and over the medial edge of the right vocal cord (Figure 2). This deposit was biopsied and resected (Figure 3).

Histological examination demonstrated laryngeal amyloidosis. The patients blood count, ESR, basic metabolic, biochemical and liver function tests were normal. The patient was subsequently referred to the NHS National Amyloidosis Centre for further exclusion of systemic disease.

1. 5. Park J, Cheng $\mathrm{H}$. Isolated laryngeal amyloidosis in a patient with a hoarse voice. Case Reports. 2013;2013(apr05 1):bcr2012008450-bcr2012008450

6. Gallivan G, Gallivan H. Laryngeal Amyloidosis Causing Hoarseness and Airway Obstruction. Journa of Voice. 2010:24(2):235-239.

\section{Discussion}

The vast majority of evidence regarding isolated laryngeal amyloidosis is in case reports highlighting its scarcity in the population. It demonstrates clinical heterogeneity with various presentations. The most common clinical presentation is an insidious onset of hoarseness. The most frequent site to be affected is the vestibular folds and ventricles and less commonly are the subglottis and aryepiglottic folds affected. In this case report we highlight progression of the disease to incomplete airway obstruction whereby amyloid deposits were identified in the subglottis and medial edge of right vocal cord. This has previously been reported in one case report in a 46 year old male with a 2-3 year protracted history of hoarseness whereby obstructing deposits were identified on the anterior commissure, vestibular and vocal folds with extension into the subglottis. The obstruction was resolved with $\mathrm{CO} 2$ laser assisted resections. ${ }^{2}$

Patients with histological diagnosis of amyloidosis require a full workup. Beyond simple tests, patients can be referred to the National Amyloidosis Center for further workup. The mainstay of treatment of the isolated form is excision of the laryngeal amyloid deposits by cold steel excision or with $\mathrm{CO} 2$ laser with regular follow up thereafter. There is no current consensus on the use of radiation therapy.

\section{Conclusion}

Laryngeal amyloidosis should be included in the differential diagnosis in the patient with stridor with an insidious background of hoarseness. Following basic screening for systemic disease, referral can be made to the National amyloidosis center for investigation of systemic disease.

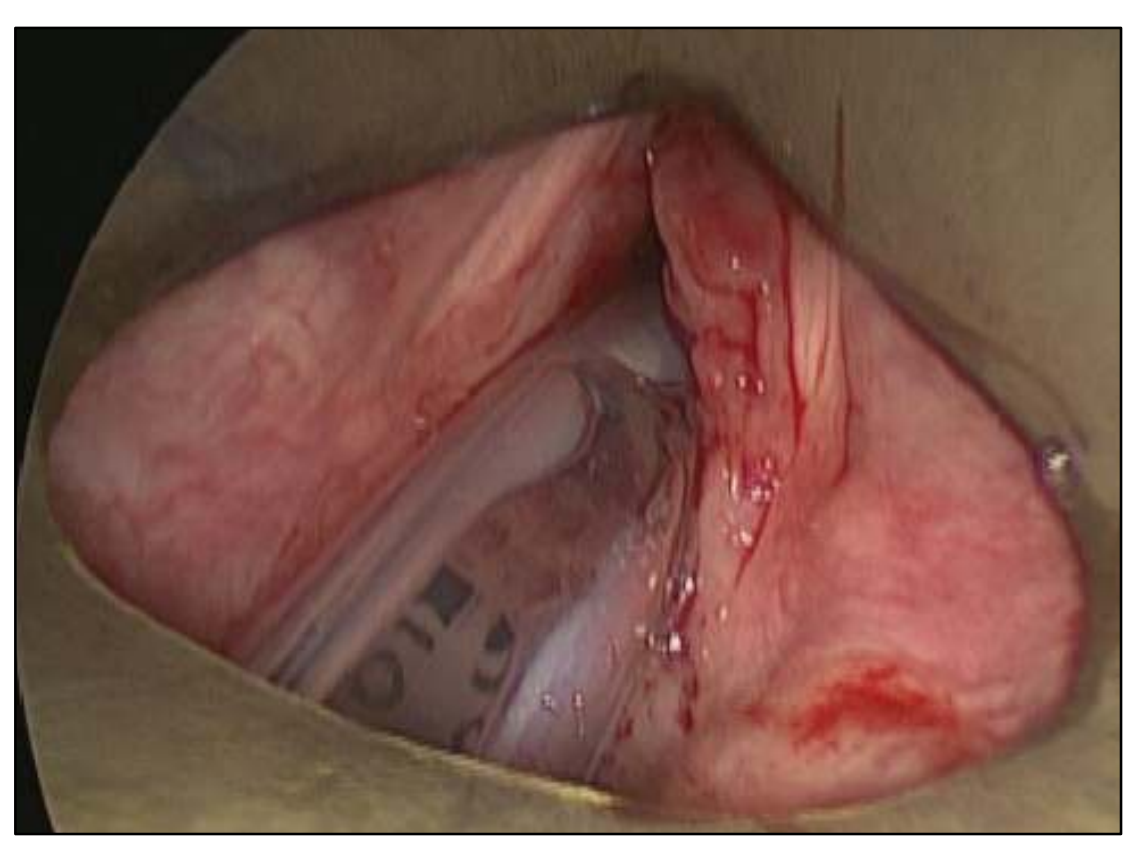

Figure 2 demonstrating a proliferative lesion medial edge right vocal cord

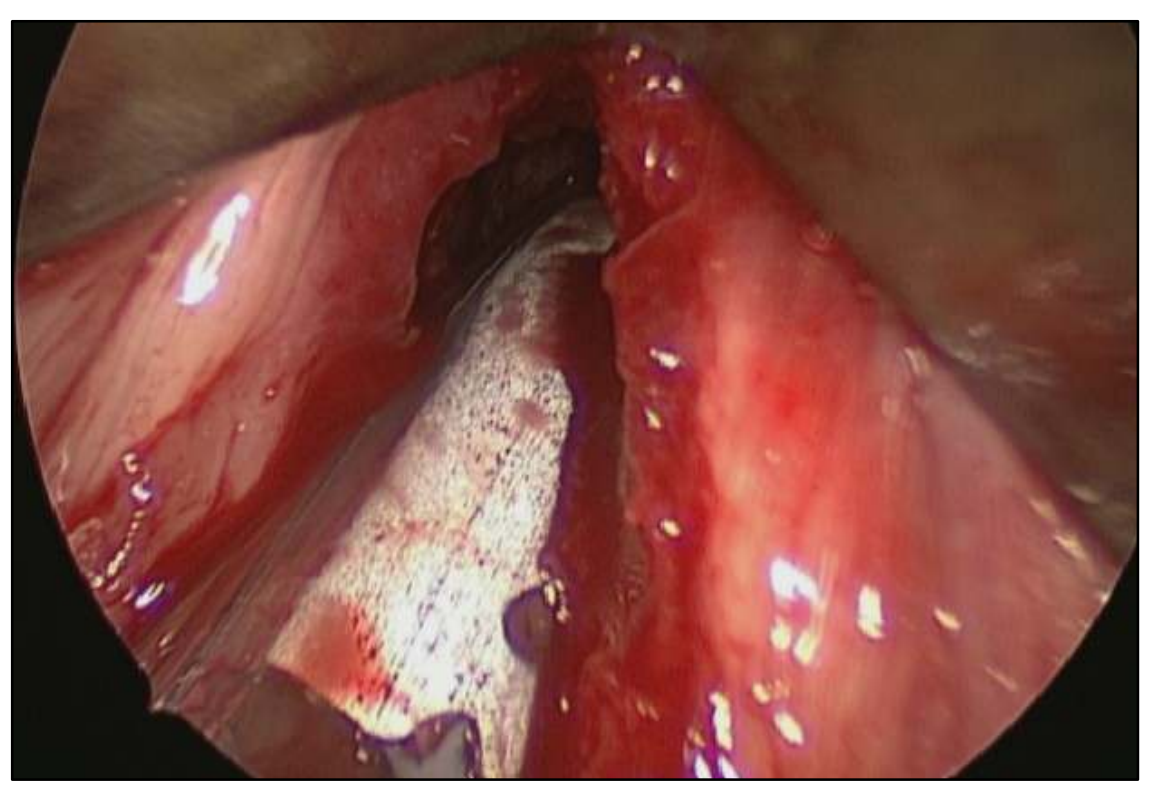

Figure 3

demonstrating appearance of vocal cord post resection 